\title{
EGF amplifies the replacement of parvalbumin-expressing striatal interneurons after ischemia
}

\author{
Tetsuyuki Teramoto, ${ }^{1}$ Jianhua Qiu, ${ }^{1}$ Jean-Christophe Plumier, ${ }^{1,2}$ \\ and Michael A. Moskowitz ${ }^{1}$ \\ ${ }^{1}$ Neuroscience Center, Radiology Department, Massachusetts General Hospital and Neuroscience Program, \\ Harvard Medical School, Boston, Massachusetts, USA \\ ${ }^{2}$ Service de Physiologie Animale, Département des Sciences de la Vie, Université de Liège, Sart-Tilman, Belgium
}

\begin{abstract}
EGF promotes proliferation and migration of stem/progenitor cells in the normal adult brain. The effect of epidermal growth factor on neurogenesis in ischemic brain is unknown, however. Here we show that intraventricular administration of EGF and albumin augments 100 -fold neuronal replacement in the injured adult mouse striatum after cerebral ischemia. Newly born immature neurons migrate into the ischemic lesion and differentiate into mature parvalbumin-expressing neurons, replacing more than $20 \%$ of the interneurons lost by 13 weeks after ischemia and representing $2 \%$ of the total BrdU-labeled cells. These data suggest that administration of EGF and albumin could be used to manipulate endogenous neurogenesis in the injured brain and to promote brain self-repair.

J. Clin. Invest. 111:1125-1132 (2003). doi:10.1172/JCI200317170.
\end{abstract}

\section{Introduction}

The subventricular zone (SVZ) lining the lateral ventricles is a source of stem cells that generate olfactory bulb interneurons throughout adulthood (1-3). EGF and its family of receptors are involved in proliferation of the adult neural stem/progenitor cells (4-9). Intraventricular infusion of EGF expands the SVZ cell population and promotes cell migration into adjacent brain parenchyma, including striatum $(7,8)$. Whether these migrating SVZ cells survive long term and give birth to differentiated neuronal populations after brain injury remains unknown.

Ischemic stroke is a particularly interesting model of brain injury because it causes selective death of neuronal subpopulations in the striatum (10-13). Spiny projection neurons containing dopamine and adenosine $3^{\prime}, 5^{\prime}$ monophosphate-regulated phosphoprotein of $32 \mathrm{KD}$ (DARPP-32 $2^{+}$and aspiny parvalbumin-containing $\left(\mathrm{PV}^{+}\right)$ interneurons die in the ischemic lesion, whereas many aspiny somatostatin-containing $\left(\mathrm{SS}^{+}\right)$or cholinergic interneurons survive (10-14). These aspiny interneurons are known to provide the bulk of the inhibitory control

Received for publication October 16, 2002, and accepted in revised form February 18, 2003.

Address correspondence to: Michael A. Moskowitz, Massachusetts General Hospital, Building 149, 13th Street, Room 6403, Charlestown, Massachusetts 02129, USA. Phone: (617) 726-8442; Fax: (617) 726-2547;

E-mail: Moskowitz@helix.mgh.harvard.edu.

Conflict of interest: The authors have declared that no conflict of interest exists.

Nonstandard abbreviations used: subventricular zone (SVZ); dopamine and adenosine $3^{\prime}, 5^{\prime}$-monophosphate-regulated phosphoprotein of $32 \mathrm{KD}$ (DARPP-32 $)$; parvalbumin (PV); somatostatin (SS); doublecortin (DCX); glial fibrillary acidic protein (GFAP); neuronal nuclear antigen (NeuN); striatal volume $\left(\mathrm{ST}_{\mathrm{V}}\right)$; neuronal replacement index $(\mathrm{NRI})$; nerve growth factor (NGF). of spiny projection neurons (15), and the disruption of the striatal neuronal circuits after stroke is considered to cause functional deficits. It is tempting to speculate that a role for adult neurogenesis would be to compensate for the loss of dead neurons and favor differentiation into specific neurons, i.e., in the case of ischemic striatum, in spiny projection and $\mathrm{PV}^{+}$neurons rather than $\mathrm{SS}^{+}$neurons. Indeed, it was reported recently that a small number of endogenous precursor cells differentiate into DARPP- $32^{+}$projection neurons within the ischemic striatum (16). The number of neurons replaced at 6 weeks after ischemia remains small, however $(0.2 \%$ of the number of neurons lost after ischemia). Clearly, these results demonstrate that endogenous neural stem cells produce very incomplete replacement of dying neurons in cases of severe injury (17). In the present study, we asked two fundamental questions: Could EGF increase neurogenesis in the injured brain, and if so, would EGF promote the replacement of both interneurons and projection neurons?

Here we show that in the ischemic striatum, administration of EGF in an albumin-containing vehicle for 1 week increases neuronal replacement 100-fold compared with vehicle. Intraventricular infusion of EGF with albumin increased the population of SVZ cells that colabeled with BrdU and doublecortin, indicating that EGF increases the number of neuronal precursor cells that eventually migrate into the ischemic lesion and differentiate into neurons. Sixtyfive percent of these newborn striatal neurons matured into $\mathrm{PV}^{+}$interneurons. Most importantly, more than $20 \%$ of $\mathrm{PV}^{+}$neurons lost after ischemia were replaced in the group treated with EGF. Our results suggest that neuronal replacement can be manipulated by exogenous growth factors such as EGF to achieve significant restoration of neuronal subpopulations lost after ischemic injury. 


\section{Methods}

Animals and surgery. Animal care and experimental protocols complied with The Principles of Laboratory Animal Care (NIH's Guide for the Care and Use of Laboratory Animals). Ten-week-old male 129S6/SvEvTac mice (Taconic Farms, Germantown, New York, USA) were anesthetized with 3\% isoflurane and maintained on $1-1.5 \%$ isoflurane in $70 \% \mathrm{~N}_{2} \mathrm{O}$ and $30 \% \mathrm{O}_{2}$ using a Fluotec 3 vaporizer (Colonial Medical Supply, Amherst, New Hampshire, USA). Regional cerebral blood flow was measured by laser-Doppler flowmetry (PF2B; Perimed, Stockholm, USA). The left MCA was occluded with an 8-0 nylon monofilament (Ethicon Inc., Somerville, New Jersey, USA) coated with a mixture of silicone resin (Xantopren; Bayer Dental Nippon KK, Osaka, Japan) and a hardener (Elastomer Activator; Bayer Dental Nippon $\mathrm{KK}$ ), as described (18). Twenty minutes later, the filament was withdrawn, and reperfusion was confirmed by laser-Doppler flowmetry. Neuronal loss was restricted to the ipsilateral striatum (Figure 1a). In a control group, mice were subjected to surgery, but the nylon filament was not introduced into the carotid artery.

EGF (human recombinant EGF; Sigma-Aldrich, St. Louis, Missouri, USA) was diluted in PBS containing BSA (Sigma-Aldrich) and unilaterally infused into the lateral ventricle using an osmotic pump (1007D; Alza Corp., Mountain View, California, USA) attached to a cannulae (brain infusion kit II; Alza Corp.) implanted at $1.4 \mathrm{~mm}$ lateral and $0.6 \mathrm{~mm}$ caudal to bregma to a depth of $1.8 \mathrm{~mm}$ from the dura. Albumin was added as a carrier upon recommendation of the EGF manufacturer and added at a concentration of $1 \mathrm{mg} / \mathrm{ml}$, consistent with recent reports studying neurogenesis $(7,8,19,20)$. EGF was infused 2 days after ischemia for 7 days at a flow rate of $0.5 \mu \mathrm{l} / \mathrm{h}$ with an initial intrapump concentration of 33 or $3.3 \mu \mathrm{g} / \mathrm{ml}$ ( 400 or $40 \mathrm{ng} / \mathrm{d}$ ). Therefore, albumin was in 3 - and 30-fold molar excess compared with EGF (400 $\mathrm{ng} / \mathrm{d}$ ) and EGF (40 ng/d), respectively. In another set of experiments, EGF (400 ng/d) was infused 21 days after ischemia for 7 days. In that group, cell proliferation was assessed on day 28 and doublecortin (DCX) and glial fibrillary acidic protein (GFAP) immunohistochemistry was performed 5 weeks after the end of the infusion (8 weeks after ischemia). The vehicle solution was sterile PBS containing $1 \mathrm{mg} / \mathrm{ml}$ BSA.

$B r d U$ injections. Animals received intraperitoneal injections of BrdU (Sigma-Aldrich; $50 \mathrm{mg} / \mathrm{kg}$; dissolved in sterile PBS and $0.007 \mathrm{~N} \mathrm{HCl}$ and filtered at $0.22 \mu \mathrm{m})$. Twice daily injections were given on days 7 and 8 after brain injury.

Immunohistochemistry. For histological evaluation, the animals were perfused transcardially with $4 \%$ paraformaldehyde in PBS under deep anesthesia. Immunohistochemistry was performed on free-floating $40-\mu \mathrm{m}$ coronal sections, as reported previously $(8,18)$. The sections were stained using the following antibodies and dilutions: anti-BrdU (1:400; Becton Dickinson Immunocytometry Systems, San Jose, California, USA), 1:400; Accurate Chemical \& Scientific Corp., Westbury, New
York, USA), anti-neuronal nuclear antigen (NeuN) (1:400; Chemicon International, Temecula, California, USA), anti-nestin (1:500; PharMingen, San Diego, California, USA), anti-EGF receptor (1:200; Santa Cruz Biotechnology Inc., Santa Cruz, California, USA), antiDCX (1:400; Santa Cruz Biotechnology Inc.), anti-Tuj1 (1:500; Covance Research Products Inc., Berkeley, California, USA), anti-GFAP (1:500; Sigma-Aldrich), anti-PV (1:500; Chemicon International), anti-DARPP-32 (1:100, Santa Cruz Biotechnology Inc.), anti-SS (1:200; Santa Cruz Biotechnology Inc.), biotinylated anti-mouse IgG (1:200; Vector Laboratories, Burlingame, California, USA), avidin-biotin-peroxidase complex (1:200; Vectastain Elite; Vector Laboratories), and secondary antibodies conjugated with cy 2 , cy5, or rhodamine red-X (1:400; Jackson ImmunoResearch Laboratories Inc., West Grove, Pennsylvania, USA). Anti-NeuN antibodies labeled with cy5 were prepared according to the manufacturer's procedure (FluroLink mAb cy5 labeling kit; Amersham International, Amersham, United Kingdom).

Quantification. All analyses were accomplished with stereologic counting methods. A systematic random sampling of every 12 th in a series of $40-\mu \mathrm{m}$ coronal sections was prepared from each animal and processed for immunohistochemistry. The striatum evaluated in this study was focused on the region from bregma $0.3-1.3$ $\mathrm{mm}$, since infarct area in the striatum ipsilateral to the insult was constant within the focused region in our pilot study. Counting of $\mathrm{BrdU}^{+}$or $\mathrm{PV}^{+}$cells was performed in the striatum in three sections per animal using a $\times 40$ objective lens. The striatum was divided into two regions by a centerline as medial and lateral part. The areas of the striatum on each section were measured on the adjacent sections stained with cresyl violet using an MCID Imaging Research image analysis system (Imaging Research Inc., St. Catherines, Ontario, Canada). The striatal volume $\left(\mathrm{ST}_{\mathrm{V}}\right)$ after ischemia was measured in the striatum in a hemisphere within bregma $0.3-1.3 \mathrm{~mm}$ by the image analysis using the Cavalieri technique (21). In this study, there was not significant difference in the $\mathrm{ST}_{V}$ between vehicle- and EGF-treated groups, although the $\mathrm{ST}_{\mathrm{V}}$ decreased during the period between 9 days and 13 weeks following ischemia (data not shown). The number of $\mathrm{BrdU}^{+}$or $\mathrm{PV}^{+}$cells within the $\mathrm{ST}_{\mathrm{V}}$ then was calculated by multiplying volume density (cells per millimeter cubed) of the labeled cells by the $\mathrm{ST}_{\mathrm{V}}$.

Counting of $\mathrm{BrdU}^{+}$cells coexpressing DCX, NeuN, or PV was performed using a Nikon TE 400 microscope with a laser confocal scanning system MRC 1024 (BioRad Laboratories Ltd., Wattford, United Kingdom). For each animal, the number of double-labeled cells was counted in the ipsilateral striatum using three sections as described above. Cells were determined double labeled when BrdU and DCX or NeuN immunoreactivity overlapped in at least five levels through a section ( $z$-step, 1 $\mu \mathrm{m})$. Neuronal replacement index (NRI) was obtained by determining the ratio between the number of newly born neurons present in the striatum $\left(\mathrm{BrdU}^{+} \mathrm{PV}^{+}\right.$cells) and the estimate of the number of dead $\mathrm{PV}^{+}$neurons. The latter 
was determined by estimating the number of $\mathrm{PV}^{+}$cells in the contralateral striatum and subtracting it from the number of $\mathrm{PV}^{+}$cells in the ischemic striatum (excluding the newly born $\mathrm{PV}^{+}$cells). The final equation is expressed as a percentage according to the following formula: $\mathrm{NRI}=100 \times\left(\right.$ number of $\mathrm{BrdU}^{+} \mathrm{PV}^{+}$cells in ipsilateral striatum) / (total number of $\mathrm{PV}^{+}$cells in contralateral striatum) - [(total number of $\mathrm{PV}^{+}$cells in ipsilateral striatum) - (number of $\mathrm{BrdU}^{+} \mathrm{PV}^{+}$cells in ipsilateral striatum)] (\%). Statistical analyses. All values are expressed as mean plus or minus SD. Mann-Whitney test or unpaired Student $t$ tests in StatView 5.0 for Macintosh were used for statistical analysis throughout the study. Dose responsiveness of EGF was evaluated using regression analysis. $P$ values less than 0.05 were considered statistically significant. At least five to six animals were used for each experiment unless otherwise specified.

\section{Results}

EGF-enhanced proliferation after ischemia. To optimize the timing of EGF infusion, we examined EGF receptor expression in neural progenitor cells in SVZ following 20 min of middle cerebral artery occlusion. Neural progenitor cells were defined as SVZ cells expressing nestin, a marker for multipotent neural stem/progenitor cells during development $(2,7)$. After focal cerebral ischemia, EGF receptor expression in the nestin ${ }^{+}$SVZ cells increased as early as 1 day (Figure 1, b and c), reached maximal expression at 3 days (Figure $1 \mathrm{~d}$ ), decreased at 9 days (not shown), and returned to basal levels at 21 days (Figure 1e). We also examined endogenous EGF expression in striatum at 1,3 , and 7 days following ischemic injury. Despite increased expression of EGF receptor in nestin ${ }^{+}$cells, we were unable to detect significant change in EGF in striatum homogenates using Western blot analysis (data not shown), suggesting that endogenous EGF levels remain low after injury (22-24).

Based on the immunohistochemical data, we started intraventricular EGF administration on day 2 , and compared the effects of 7-day infusion of EGF or vehicle on cell proliferation using BrdU incorporation into replicating DNA $(7,8)$. A low dose of EGF $(40 \mathrm{ng} /$ day) was sufficient to increase the number of $\mathrm{BrdU}^{+}$cells 11-fold in the medial striatum, but not in the lateral striatum, compared with ischemia plus vehicle (Figure $1 \mathrm{j}$ ). Moreover, increasing EGF dose to $400 \mathrm{ng} /$ day further increased the number of $\mathrm{BrdU}^{+}$cells 1.5 -fold in the medial striatum (17-fold increase compared with ischemia
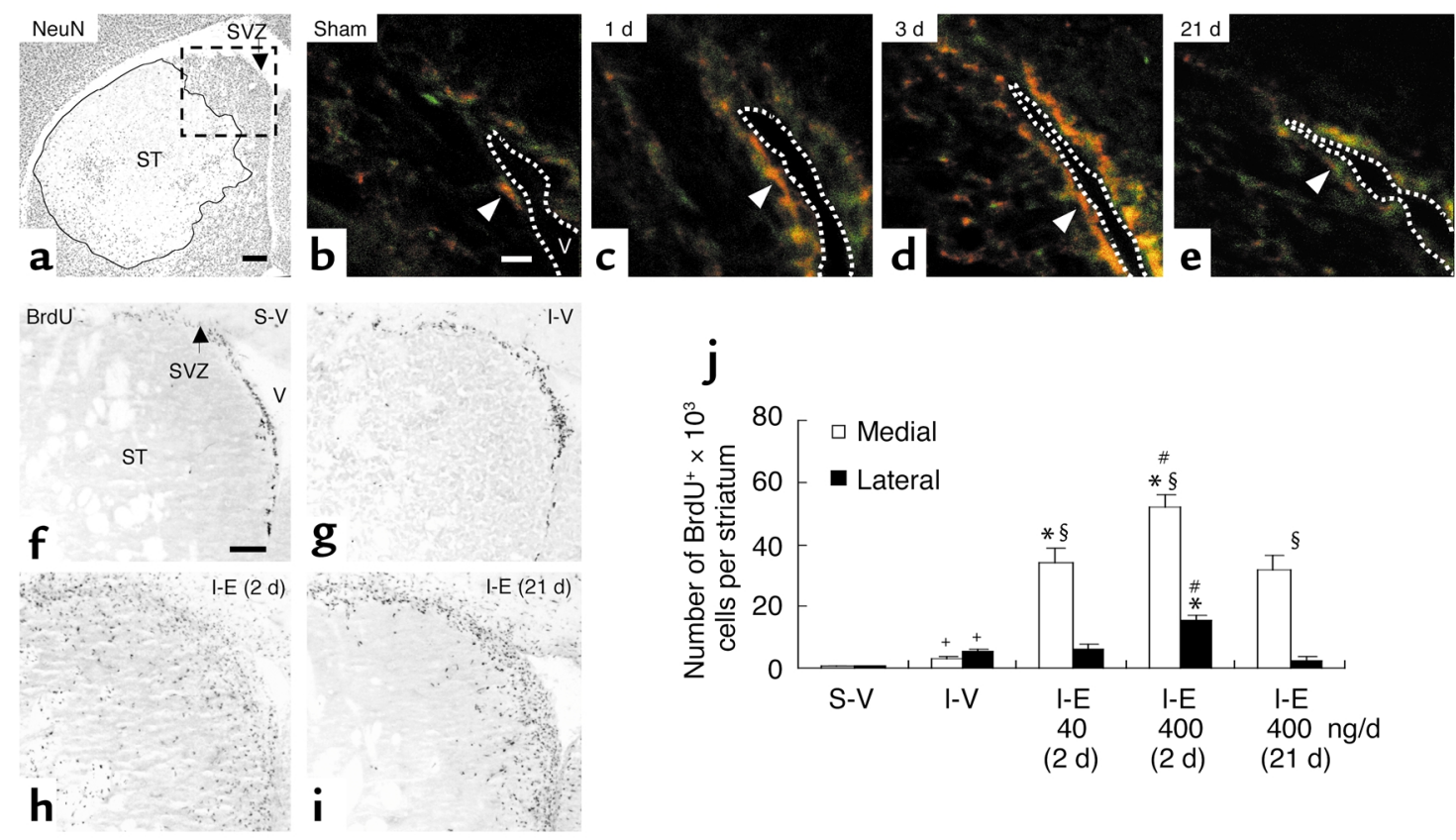

\section{Figure 1}

EGF-enhanced proliferation after ischemia. (a) Ischemia reperfusion caused a marked loss of $\mathrm{NeuN}^{+}$neurons (outlined area) confined to the striatum (ST). SVZ, subventricular zone. Scale bar is $200 \mu \mathrm{m}$. (b-e) Without ischemia (b), few nestin ${ }^{+}$cells (green) expressing EGF receptors (red) surrounded the lateral ventricle $(\mathrm{V})$. After ischemia, the intensity of EGF receptor immunoreactivity in nestin ${ }^{+} \mathrm{SVZ}$ cells (arrowheads) and the number of nestin ${ }^{+}$cells expressing EGF receptor increased at 1 day (c), reached maximum at 3 days (d), and returned to basal levels at 21 days (e). The dotted line delineates lateral ventricle. Scale bar is $20 \mu \mathrm{m}\left(n=3-4 /\right.$ time-point). (f-i) BrdU ${ }^{+}$cells at 9 days after sham or ischemia in an area corresponding to the box (a). After vehicle, the number of BrdU ${ }^{+}$cells was higher after ischemia $(\mathbf{g})$ than sham (f) (quantification in $\mathbf{j}$ ). Ischemia followed by EGF (I-E; $400 \mathrm{ng} /$ day) increased BrdU ${ }^{+}$cells and was greater when EGF infusion started 2 days (h) rather than 21 days (i) after ischemia. (f) Scale bar is $100 \mu \mathrm{m}$. Bregma, $0.8 \mathrm{~mm}$. (j) Quantification. S-V, sham-operated; $\mathrm{I}-\mathrm{V}$, ischemic group with vehicle; I-E (2d), ischemic groups with EGF (400 ng/day) initiated at day 2; I-E (21d), day 21 after ischemia. The effects of EGF (40 and $400 \mathrm{ng} /$ day) initiated at day 2 were compared. +Significant difference between S-V and I-V; *between vehicle- and EGF-treated ischemic groups; "between groups treated with EGF 2 days or 21 days; §between medial and lateral striatum within the same group; $n=3-4 /$ group, unpaired Student $t$ test, $P<0.05$. 

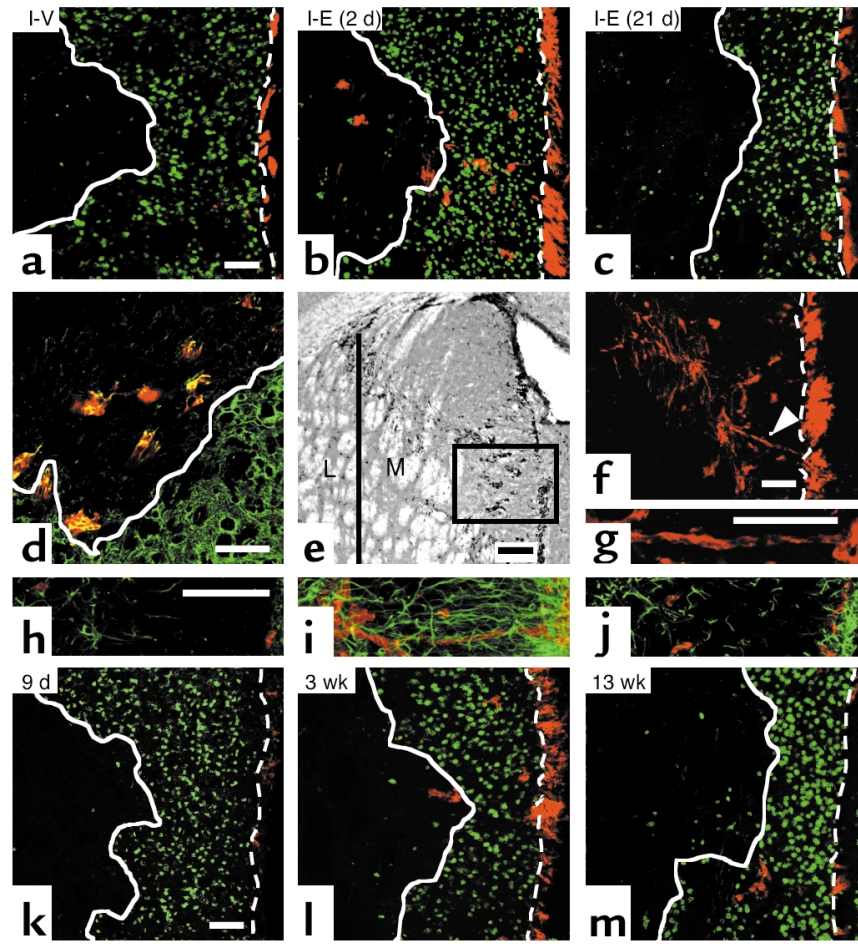

Figure 2

Migrating neuroblasts after EGF treatment. (a) Using confocal microscopy, 5 weeks after ischemia, very few $D^{2} X^{+}$cells (red) were observed in the striatal lesion (white solid line) after vehicle. $\mathrm{DCX}^{+}$cells dramatically increased by EGF infusion initiated on day 2 (b) but not on day 21 (c) ( $n=3-4$ per group; for quantification see Table 1$)$, revealing the importance of EGF signaling for cell migration early after ischemia. (d) Colocalization of DCX (red) and Tuj1 (green) confirmed their phenotype. (e) $\mathrm{DCX}^{+}$cells were predominantly within medial $(M)$ relative to lateral $(\mathrm{L})$ striatum. ( $\mathbf{f}$ and $\mathbf{g}$ ) Chains or clusters of $\mathrm{DCX}^{+}$(red) cells (arrowhead, magnified in $\mathbf{g}$ ) connecting SVZ and the striatal lesion were observed in medial striatum (e). (h-j) GFAP+ glial cells (green) and $\mathrm{DCX}^{+}$neuroblasts (red) between SVZ (right end) and the damaged striatum (left side) were rare in vehicle (h) or with EGF administration initiated at day 21 (j), compared with EGF at day 2 post-ischemia (i). Note the orientation of GFAP ${ }^{+}$ processes in (i). (k-m) The spatial distribution and number of $\mathrm{DCX}^{+}$(red) cells changed with time after EGF. DCX+ cells were few at the end of the infusion period $(\mathbf{k})$ and progressively increased in the area devoid of $\mathrm{NeuN}^{+}$ cells (green) at 3 weeks (I) and 5 weeks (b). By 13 weeks, DCX+ were mostly outside the lesion $(\mathbf{m})$, suggesting that $\mathrm{DCX}^{+}$cells may have differentiated into mature neurons. Scale bar is $50 \mu \mathrm{m}$ except for $(\mathbf{e}), 100 \mu \mathrm{m}$. Breg$\mathrm{ma}, 0.8 \mathrm{~mm}$. Dotted line, the border between SVZ and striatum.

plus vehicle), revealing a dose dependency following EGF infusion $(P<0.05)$. Delayed administration of EGF (400 $\mathrm{ng} /$ day beginning at 21 days after ischemia) was less effective, resulting in a 1.6-fold decrease in $\mathrm{BrdU}^{+}$cells in the medial striatum, compared with ischemia plus EGF ( $400 \mathrm{ng} /$ day initiated at 2 days after ischemia) (Figure 1, $i$ and $j$ ). These data suggest the importance of EGF-EGF receptor signaling in cell proliferation early after stroke. Migrating neuroblasts after EGF treatment. Five weeks after ischemia, immature migrating neuroblasts labeled with $\operatorname{DCX}(16,25)$ were found throughout the medial striatum when EGF (400 ng/day) infusion was initiated on day 2, but not on day 21 (Figure 2, b and c). In EGF (400 $\mathrm{ng} /$ day initiated at day 2 after ischemia), $\mathrm{DCX}^{+}$cells were colabeled with a neuronal marker, Tuj1(2), confirming their neuronal phenotype (Figure 2d). The number of migrating neuroblasts was high in the medial striatum adjacent to SVZ (Figure 2e). Chains (and clusters) of $\mathrm{DCX}^{+}$migrating neuroblasts appeared to link the SVZ and the striatal lesion and were observed in the treated brain (Figure 2, f and g). Strikingly, EGF infusion also increased $\mathrm{GFAP}^{+}$cells exhibiting long processes following ischemia (Figure 2i). Moreover, $\mathrm{GFAP}^{+}$cells and their processes organized a network to link SVZ and striatal lesion. This network was absent when EGF infusion was delayed after ischemia (Figure 2j). These observations suggest that a network of $\mathrm{GFAP}^{+}$cells could participate in the migration of $\mathrm{DCX}^{+}$cells toward the ischemic lesion and possibly for trophic support or as a lattice for neuroblast migration (26-28). Although our observations also suggest that neuroblasts within the striatal lesion originated from the SVZ as reported previously (16), however, our data did not exclude the possibility that neuroblasts also arose from resident precursors or circulating stem cells $(4,5,29)$. At 3 and 5 weeks after ischemia, however, many $\mathrm{DCX}^{+}$neuroblasts were found outside the SVZ (Figure 2, b and l) with a gradient of cells from SVZ to the lesion. These findings are consistent with previous reports showing that EGF infusion promotes division of precursors (also referred to as SVZ type C cells expressing EGF receptor) into neuroblasts (19) as well as migration of SVZ cells into striatum (7). Taken together, the data suggest that migrating neuroblasts within the ischemic striatum derived from SVZ progenitors cells in response to EGF.

Neuronal differentiation in the ischemic striatum. At 13 weeks, the number of $\mathrm{DCX}^{+}$plus BrdU cells decreased in the lesion (Figure $2 \mathrm{~m}$ ) but not in the intact area adjacent to the SVZ. Hence, $\mathrm{DCX}^{+}$neuroblasts within the ischemic lesion either express markers of mature neurons after losing their $\mathrm{DCX}^{+}$phenotype or die.

To elucidate whether immature neuroblasts differentiate into mature neurons, we compared the distribution and the number of $\mathrm{BrdU}^{+}$cells expressing DCX or NeuN (mature neuronal marker) between 5 and 13 weeks after ischemia using twocolor confocal imaging with serial sections in the $z$ plane. BrdU ${ }^{+}$striatal neurons expressing NeuN were detected mainly at the boundary of the ischemic lesion in medial striatum (Figure 3). In fact, there were 13-fold more $\mathrm{BrdU}^{+} \mathrm{NeuN}^{+}$cells in the medial striatum than in the lateral striatum at 13 weeks $(P<0.01$, data not shown). A similar proportion was observed for $\mathrm{DCX}^{+}$ neuroblasts at 5 weeks (Figure 2). In addition, ischemia and EGF administration augmented the absolute number of $\mathrm{BrdU}^{+} \mathrm{NeuN}^{+}$cells with time (Table 1). The ratio of $\mathrm{BrdU}^{+} \mathrm{NeuN}^{+}$neurons to total $\mathrm{BrdU}^{+}$cells increased from $1.1 \%$ to $3.0 \%$ between 5 and 13 weeks (Table 1 ), 

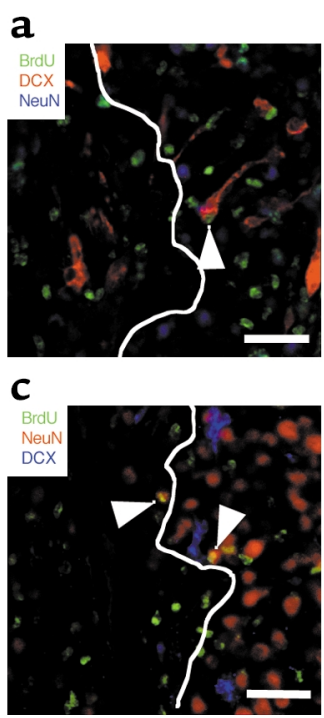

b
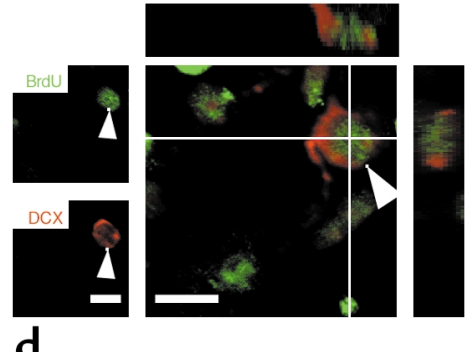

d

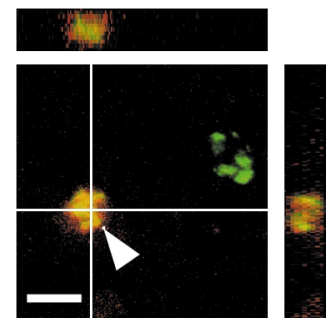

Figure 3

Neuronal differentiation in the ischemic striatum. (a and $\mathbf{c}$ ) Representative confocal microscopic images of BrdU, DCX, and NeuN colabeling showing that BrdU-labeled neurons (arrowheads) were located in the boundary zone of the ischemic lesion at 5 (a) and 13 (c) weeks. A white solid line delineates the lesion area. Scale bar is 50 $\mu \mathrm{m}$. (b and $\mathbf{d}$ ) Confocal 3D analyses of BrdU ${ }^{+} D C X^{+}$cells at 5 weeks (b) and $\mathrm{BrdU}^{+} \mathrm{NeuN}^{+}$cells at 13 weeks (d) after ischemia. Reconstructed orthogonal images are presented as viewed from the sides in both the $x-z$ (top) and $y-z$ (right) planes. Arrowheads indicate double-labeled cells. Scale bar is $10 \mu \mathrm{m} . n=5-6$ per group (for quantification, see Table 1).

whereas the number of $\mathrm{DCX}^{+}$cells decreased (Figure 2, $\mathrm{b}$ and $\mathrm{m}$; Table 1). The distribution and the appearance of mature neurons with time, taken together with a decrease in the number of immature cells, suggested that $\mathrm{DCX}^{+}$neuroblasts differentiated into mature $\mathrm{NeuN}^{+}$neurons within the ischemic striatum. Some $\mathrm{BrdU}^{+} \mathrm{DCX}^{+}$cells may have died as well, as reflected by the decrease in $\mathrm{BrdU}^{+} \mathrm{DCX}^{+}$cells that became NeuN positive from week 5 to 13 (Table 1).

The use of $\mathrm{BrdU}^{+}$incorporation as a marker of cell division and newly born neurons requires caution in the injured brain because cells with DNA damage also incorporate $\mathrm{BrdU}$ in the repair process (30). However, the $S V Z$ is remote from the ischemic lesion, and its cells are unlikely to sustain DNA damage (31). Moreover, two results suggest that BrdU incorporation reflected division of progenitor cells in our experiments: (a) we did not detect TUNEL staining in $\mathrm{NeuN}^{+}$cells at 9 days after ischemia (data not shown), and (b) there were no $\mathrm{BrdU}^{+} \mathrm{NeuN}{ }^{+}$double-labeled cells in the striatum at 9 days after ischemia (peak of $\mathrm{BrdU}^{+}$cell number). Taken together, these results confirmed that $\mathrm{BrdU}^{+} \mathrm{NeuN}^{+}$ cells were not injured neurons that incorporated BrdU as part of their DNA repair process, but were striatal neurons differentiated from newly generated precursor cells at 5 and 13 weeks after injury.

Phenotypes of the newly born neurons. We next determined whether newly born cells were differentiated into specific neuronal phenotypes. Hence, we characterized $\mathrm{BrdU}^{+} \mathrm{NeuN}^{+}$neurons by immunohistochemistry using PV-, SS-, and DARPP-32-specific antibodies. At 13 weeks after ischemia, numerous $\mathrm{BrdU}^{+} \mathrm{NeuN}^{+}$ striatal cells expressing PV were detected (Figure 4a) at the boundary zone of the ischemic lesion (Figure 4e), where new mature neurons were detected (Figure 3c). In fact, there were $345 \pm 69 \mathrm{BrdU}^{+} \mathrm{NeuN}^{+} \mathrm{PV}^{+}$cells from a total of $525 \pm 83 \mathrm{BrdU}^{+} \mathrm{NeuN}^{+}$cells (Figure 4f; Table 1 ) in the medial striatum, indicating that $65 \%$ of newly born neurons differentiated into $\mathrm{PV}^{+}$interneurons. The remaining BrdU $\mathrm{NeuN}^{+}$cells did not express either SS or DARPP-32 (Figure 4e), and their identity remains unknown. Similar results were observed in the lateral striatum although the overall number of BrdU-positive neurons was much lower (data not shown). EGF increases neuronal replacement of lost $\mathrm{PV}^{+}$neurons 100 -fold $(21 \% \pm 4 \%$ versus $0.2 \% \pm 0.5 \%$ for vehicle administration; Figure 4g), corresponding to a restoration of the $\mathrm{PV}^{+}$population to $60 \%$ of its number before injury (Figure $4 \mathrm{~h}$ ). $\mathrm{PV}^{+}$interneurons normally extend a rich network of dendrites (32). Indeed, a rich network of $\mathrm{PV}^{+}$dendrites was visualized within injured striatum (Figure 4a), demonstrating that these newly born neurons also exhibit the morphological characteristics of aspiny $\mathrm{PV}^{+}$neurons. If the dendrites of these newly born $\mathrm{PV}^{+}$cells also contact 100 projection neurons, as

Table 1

Numbers of newborn neurons in medial striatum at 5 and 13 weeks after ischemia

\begin{tabular}{|c|c|c|c|c|c|c|}
\hline Group & Weeks after ischemia & (a) Total BrdU ${ }^{+}$cells & (b) $\mathrm{BrdU}^{+} \mathrm{DCX}^{+}$cells & $\%$ of $(a) /(b)$ & (c) $\mathrm{BrdU}^{+} \mathrm{NeuN}^{+}$cells & $\%$ of $(c) /(a)$ \\
\hline \multirow[t]{2}{*}{ S-V } & 5 & $317 \pm 56$ & Ud & - & Ud & - \\
\hline & 13 & $275 \pm 64$ & Ud & - & Ud & - \\
\hline \multirow[t]{2}{*}{$S-E$} & 5 & $16,005 \pm 2,178$ & $120 \pm 84$ & $0.7 \pm 0.5$ & Ud & - \\
\hline & 13 & $12,325 \pm 1,893^{\mathrm{D}}$ & $75 \pm 50$ & $0.6 \pm 0.4$ & Ud & - \\
\hline \multirow[t]{2}{*}{ I-V } & 5 & $1,182 \pm 253$ & $20 \pm 21$ & $1.6 \pm 1.8$ & $3 \pm 3$ & $0.3 \pm 0.3$ \\
\hline & 13 & $913 \pm 227$ & Ud & - & $8 \pm 13$ & $0.8 \pm 1.3$ \\
\hline \multirow[t]{2}{*}{ I-E } & 5 & $22,027 \pm 4,378$ & $815 \pm 188^{A}$ & $3.7 \pm 0.3$ & $245 \pm 134^{\mathrm{A}}$ & $1.1 \pm 0.6$ \\
\hline & 13 & $17,505 \pm 2,943$ & $110 \pm 34^{B, C}$ & $0.7 \pm 0.3^{C}$ & $525 \pm 83^{B}, C$ & $3.0 \pm 0.3$ \\
\hline
\end{tabular}

Infusion of EGF ( $400 \mathrm{ng} /$ day) was initiated on day 2 after ischemia. Data correspond to number of cells per striatum and are expressed as mean \pm S.D. ( $n=5-6 /$ group). Ud, undetected; S-V, sham-operated group with vehicle treatment; S-E, sham-operated group with EGF; I-V, ischemic group with vehicle; $\mathrm{I}$-E, ischemic group with EGF. ${ }^{A} P<0.01$ vs. I-V at 5 weeks; ${ }^{B} P<0.01$ vs. I-V at 13 weeks; ${ }^{C} P<0.05$ vs. I-E at 5 weeks; ${ }^{D} P<0.05$ vs. S-E at 5 weeks, unpaired $t$ test. 

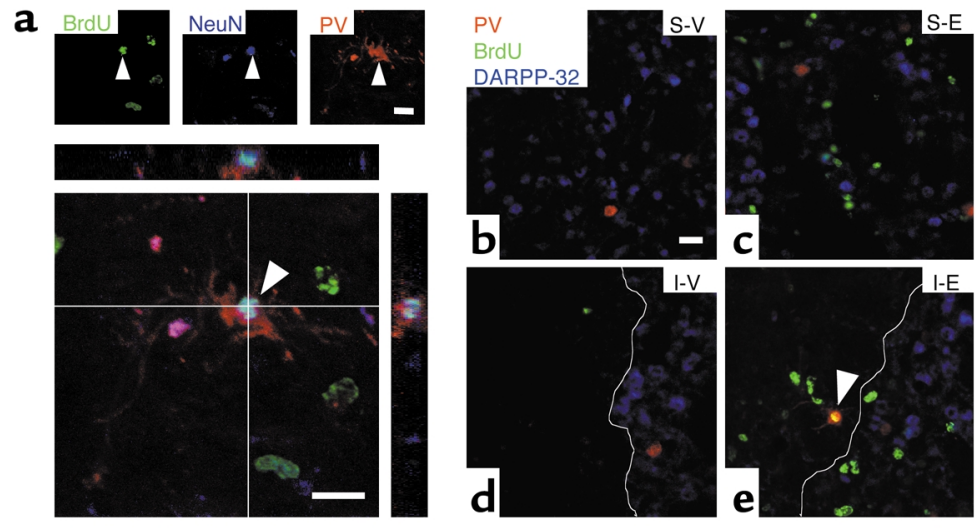

f

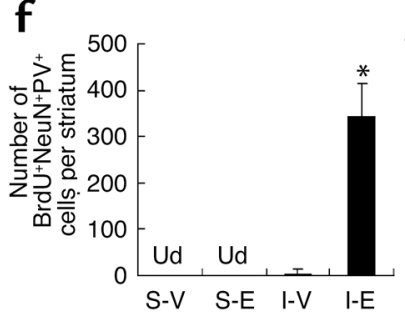

$\mathbf{g}_{30}$

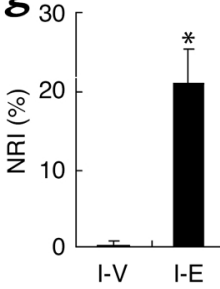

h

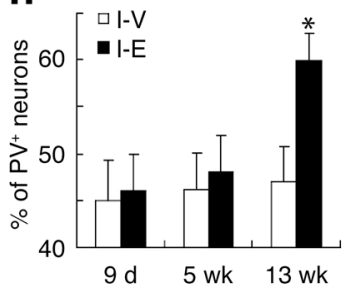

Figure 4

Neuronal replacement in the ischemic striatum. (a) Confocal 3D reconstruction revealed newborn $\mathrm{PV}^{+}$neurons (arrowhead) triple-labeled with $\mathrm{BrdU}, \mathrm{NeuN}$, and $P V$ in the medial striatum of EGF-treated mice at 13 weeks after ischemia. (b-e) The triple-labeling with BrdU, PV, and DARPP-32 revealed that newborn $\mathrm{PV}^{+}$neurons (e, arrowhead) were located in the boundary zone of the ischemic lesion. White solid line delineates the lesion area. Scale bars are $20 \mu \mathrm{m}$. (f) Numbers of $\mathrm{BrdU}^{+} \mathrm{NeuN}^{+} \mathrm{PV}^{+}$cells in the medial striatum at 13 weeks after ischemia. Ud, undetected in VAS-V and S-E groups. (g) NRI (see text) indicates ratio between newborn neurons and the neurons lost after injury. (h) Progressive repopulation of $\mathrm{PV}^{+}$neurons in the ischemic striatum measured as a percentage of $\mathrm{PV}^{+}$neurons in ischemic striatum relative to the number in the nonischemic contralateral striatum. * Significant difference between ischemic group administered with vehicle $(\mathrm{I}-\mathrm{V})$ or EGF $(\mathrm{I}-\mathrm{E})(P<0.01) ; n=5-6 /$ group, unpaired Student $t$ test. reduced infarct volumes (34). Moreover, in the adult as well as in the developing brain, the expression of EGF receptors can stimulate proliferation and migration of neural progenitor cells within SVZ (35-37). We did not detect effects of EGF on infarct volumes (data not shown), but observed the effects of EGF infusion on neurogenesis, especially when EGF receptor expression was high in SVZ a few days after ischemia but not at 21 days after ischemia (Figure 1). These results suggest that EGF treatment promotes cell proliferation and migration through EGF receptor-mediated mechanisms.

Compared with albumin vehicle alone, the number of newly generated neuroblasts and mature neurons was drastically increased after EGF plus albumin administration (Table 1). Albumin was added to the vehicle to stabilize EGF (as recommended by Sigma-Aldrich) and to provide consistent dosing especially in view of its potential for degrading and sticking during prolonged infusion (7 days) using a pump reservoir (Alzet pump). Albumin has important biological effects in ischemia, and its role in neurogenesis has not been well explored. Albumin reduces ischemic injury and oxygen radical damage during reperfusion when administered acutely intravenously up to 24 hours after ischemia (38-40). Albumin did not reduce infarct size, however, when we infused the albumin-containing vehicle intracerebroventricularly 48 hours after ischemia (data not shown). In retrospect, we wonder does the normal cell, the potential impact of these findings to striatal function is impressive.

Injury was an important factor in the process of neuronal replacement. In its absence, EGF alone increased SVZ cell number, but did not promote maturation of neuroblasts into neurons (Table 1; Figure 4). Ischemic injury itself stimulated neuronal replacement, although the number of mature neurons remained rare (Table 1; Figure 4). Therefore, EGF receptor induction and glial activation in response to ischemia, provided crucial substrates for EGF-mediated neuronal replacement.

\section{Discussion}

Our results demonstrate that endogenous neuronal replacement can be amplified after ischemic brain injury and that EGF, only minimally expressed in ischemic striatum, can enhance the neuronal recruitment process. Early after ischemia, EGF receptor levels increased in SVZ cells and in cell types such as neurons and glia (33), and EGF receptor activation by acute administration (within 30 min of ischemia) of ligands whether the addition of albumin to the vehicle is necessary because proliferation of SVZ cells or recruitment of SVZ cells into the damaged striatum was observed after infusion of an EGF receptor ligand (heparin-binding EGF-like growth factor or TGF- $\alpha$ ) within striatum or ventricle in the absence of albumin $(41,42)$. Furthermore, EGF in the absence of albumin improved a learning response (passive avoidance) and increased SVZ cell proliferation when administered acutely to aged mice (43), although this response was observed only when EGF was coadministered with nerve growth factor (NGF). Finally, based on our own data, any possible synergistic action of albumin on neurogenesis would have to be EGF-specific because coadministration of albumin plus FGF-2 failed to promote migration of newly born neuroblasts in the ischemic striatum or differentiation into mature neurons (data not shown). Nevertheless, our experiments do not rule out the possibility that albumin enhanced EGF's action by a mechanism independent of protein stabilization, and additional experiments directed at studying albumin's effects on EGF receptor binding 
and signal transduction (as well as the use of another protein carrier) would be of interest.

Thirteen weeks after ischemia plus EGF administration, $65 \%$ of newly born neurons matured into $\mathrm{PV}^{+}$ neurons, one type of striatal GABAergic interneurons (Figure 4) $(15,44)$. In the CNS, PV and other calciumbinding proteins such as calbindin and calretinin play a vital role in calcium homeostasis and are generally thought of as calcium buffering rather than calcium regulatory proteins (45). Despite this, a specific role of parvalbumin in neuronal function is still unclear. In the $d t(s z)$ mutant hamster, however, a genetic model of a type of paroxysmal dystonia, the number of $\mathrm{PV}^{+}$ GABAergic neurons was selectively reduced and administration of $\mathrm{GABA}(\mathrm{A})$ receptor agonist muscimol improved the dystonic syndrome, supporting the importance of striatal GABAergic $\mathrm{PV}^{+}$interneurons in the control of motor function (46). Similarly, immunohistochemical analysis suggests that degeneration of $\mathrm{PV}^{+}$interneurons are involved in the pathophysiology of Huntington disease $(47,48)$. In stroke, the rescue of $\mathrm{PV}^{+}$striatal cells by BDNF partially restores functional deficits (13). These results suggest that regeneration of $\mathrm{PV}^{+}$interneurons might help functional recovery after injury and should be a part of strategies to repair striatum, together with neuronal replacement of other types of striatal interneurons and projection neurons.

Unexpectedly, neuronal differentiation after EGF administration was limited to $\mathrm{PV}^{+}$neurons. Newly born neurons did not mature into $\mathrm{SS}^{+}$-expressing interneurons, or even DARPP- $32^{+}$projection neurons, representing about $95 \%$ of the striatal neuronal population (14). This result suggests that EGF favors the expression of specific phenotypes during differentiation of adult striatal progenitors. The absence of DARPP- $32^{+}$neurons in the ischemic striatum after EGF treatment, however, contrasts with EGF-mediated in vitro differentiation of progenitor cells into projection neurons $(4,5)$ and maturation of $42 \%$ of newly born neurons into DARPP- $32^{+}$projection in the ischemic rat striatum in the absence of EGF treatment (16). The cause for these differences is still unclear. It is possible that in the ischemic brain, EGF specifically promoted maturation and differentiation of $\mathrm{PV}^{+}$neurons while possibly suppressing maturation and differentiation of DARPP- $32^{+}$neurons. In the absence of EGF treatment, our protocol for BrdU administration limited the detection of $\mathrm{BrdU}^{+}$cells after ischemia to only about 1,000 cells (Table 1 ), and of those only three to eight expressed a mature neuron phenotype $\left(\mathrm{NeuN}^{+}\right)$. Hence, it was not possible to identify the phenotype of these infrequent BrdU-NeuN-positive cells in the absence of EGF treatment. The absence of DARPP- $32^{+}$neurons after EGF treatment could also be due to species differences or severity of ischemia $(2 \mathrm{~h}$ versus $20 \mathrm{~min}$ ). Additional studies will be necessary to clarify this point and determine whether the newly born $\mathrm{PV}^{+}$cells are also GABAergic.
Ischemic injury provides important signals to encourage cell migration and differentiation since EGF infusion did not increase neurogenesis in the normal mouse striatum (Figure 4, Table 1). Similarly, administration of EGF alone or with FGF-2 did not increase neurogenesis in the injured spinal cord (49). In contrast, EGF and FGF-2 infusion promotes neuronal regeneration in the ischemic hippocampus, a region where endogenous neurogenesis occurs in the absence of injury (20). In the present study, we report that ischemia enhances EGF-mediated neurogenesis to replace neurons lost in the medial striatum, a region usually devoid of neurogenesis. By 4 weeks after EGF infusion alone, neuroblast migration was restricted to an area adjacent to the SVZ. With ischemia, neuroblasts repopulated the lesion. In addition, EGF in the absence of ischemia did not regulate neuronal differentiation. The occasional $\mathrm{DCX}^{+}$cell migrating toward the intact striatum did not differentiate into mature neurons after EGF, confirming other reports $(8,50)$. It is unclear how ischemia promotes neuroblast migration and neuronal differentiation in EGF-treated animals. An early cue could be the expression of EGF receptor on SVZ cells. Glia may also be important. We found that EGF increased the number of activated glia $\left(\mathrm{GFAP}^{+}\right)$between the SVZ and the ischemic lesion. In addition, we found that $\mathrm{GFAP}^{+}$cells tended to surround neuroblasts migrating toward the ischemic striatum at both 5 and 13 weeks after ischemia (Figure 2 ). Others reported that interactions between $\mathrm{GFAP}^{+}$ cells and neuronal precursors are necessary for neuroblast migration, differentiation, and/or survival in the adult brain $(26,28,51)$. Neurotransmitters and growth factors such as stem cell factor, GABA, and glutamate may be important for neuroblast migration and neuronal differentiation $(18,22,52,53)$, as they are during development (54-57). FGF-2 promotes neuronal differentiation of EGF-responsive progenitor cells in vitro $(35,58,59)$, and FGF-2 levels increase in striatum fivefold at 7 days after ischemia (data not shown). We did not observe any effect of FGF-2 on neuronal differentiation of newly born cells in the ischemic striatum with doses that promoted cell proliferation in SVZ (data not shown), however. These results highlight that the proliferation of SVZ cells alone is not sufficient to increase neuronal replacement within the ischemic striatum. Further studies examining the identity of specific microenvironmental cues that regulate EGF-mediated striatal neurogenesis after ischemia could provide new opportunities to promote repair in injured adult brain.

\section{Acknowledgements}

We thank S. Yoshimura, Y. Takagi, and J. Harada for their technical advice, and M.J. Whalen for editorial assistance. This work was supported by NIH Indepartmental Stroke Program Project 5 P50 NS10828 (M.A. Moskowitz) and by the American Heart Association (J.-C. Plumier). 
1. Betarbet, R., Zigova, T., Bakay, R.A., and Luskin, M.B. 1996. Dopaminergic and GABAergic interneurons of the olfactory bulb are derived from the neonatal subventricular zone. Int. J. Dev. Neurosci. 14:921-930.

2. Chiasson, B.J., Tropepe, V., Morshead, C.M., and van der Kooy, D. 1999. Adult mammalian forebrain ependymal and subependymal cells demonstrate proliferative potential, but only subependymal cells have neural stem cell characteristics. J. Neurosci. 19:4462-4471.

3. Alvarez-Buylla, A., and Garcia-Verdugo, J.M. 2002. Neurogenesis in adult subventricular zone. J. Neurosci. 22:629-634.

4. Reynolds, B.A., Tetzlaff, W., and Weiss, S. 1992. A multipotent EGFresponsive striatal embryonic progenitor cell produces neurons and astrocytes. J. Neurosci. 12:4565-4574.

5. Reynolds, B.A., and Weiss, S. 1992. Generation of neurons and astrocytes from isolated cells of the adult mammalian central nervous system. Science. 255:1707-1710.

6. Seroogy, K.B., Gall, C.M., Lee, D.C., and Kornblum, H.I. 1995. Proliferative zones of postnatal rat brain express epidermal growth factor receptor mRNA. Brain Res. 670:157-164.

7. Craig, C.G., et al. 1996. In vivo growth factor expansion of endogenous subependymal neural precursor cell populations in the adult mouse brain. J. Neurosci. 16:2649-2658.

8. Kuhn, H.G., Winkler, J., Kempermann, G., Thal, L.J., and Gage, F.H. 1997. Epidermal growth factor and fibroblast growth factor- 2 have different effects on neural progenitors in the adult rat brain. J. Neurosci. 17:5820-5829

9. Gritti, A., et al. 1999. Epidermal and fibroblast growth factors behave as mitogenic regulators for a single multipotent stem cell-like population from the subventricular region of the adult mouse forebrain. J. Neurosci. 19:3287-3297.

10. Chesselet, M.F., Gonzales, C., Lin, C.S., Polsky, K., and Jin, B.K. 1990. Ischemic damage in the striatum of adult gerbils: relative sparing of somatostatinergic and cholinergic interneurons contrasts with loss of efferent neurons. Exp. Neurol. 110:209-218.

11. Chen, Q., Veenman, C.L., and Reiner, A. 1996. Cellular expression of ionotropic glutamate receptor subunits on specific striatal neuron types and its implication for striatal vulnerability in glutamate receptor-mediated excitotoxicity. Neuroscience. 73:715-731.

12. Andsberg, G., Kokaia, Z., Bjorklund, A., Lindvall, O., and Martinez-Serrano, A. 1998. Amelioration of ischaemia-induced neuronal death in the rat striatum by NGF-secreting neural stem cells. Eur. J. Neurosci. 10:2026-2036

13. Andsberg, G., et al. 2002. Neuropathological and behavioral consequences of adeno-associated viral vector-mediated continuous intrastriatal neurotrophin delivery in a focal ischemia model in rats. Neurobiol. Dis. 9:187-204.

14. Anderson, K.D., and Reiner, A. 1991. Immunohistochemical localization of DARPP-32 in striatal projection neurons and striatal interneurons: implications for the localization of D1-like dopamine receptors on different types of striatal neurons. Brain Res. 568:235-243.

15. Kawaguchi, Y. 1997. Neostriatal cell subtypes and their functional roles. Neurosci. Res. 27:1-8.

16. Arvidsson, A., Collin, T., Kirik, D., Kokaia, Z., and Lindvall, O. 2002. Neuronal replacement from endogenous precursors in the adult brain after stroke. Nat. Med. 8:963-970.

17. Horner, P.J., and Gage, F.H. 2000. Regenerating the damaged central nervous system. Nature. 407:963-970.

18. Yoshimura, S., et al. 2001. FGF-2 regulation of neurogenesis in adult hippocampus after brain injury. Proc. Natl. Acad. Sci. U. S. A. 98:5874-5879.

19. Doetsch, F., Petreanu, L., Caille, I., Garcia-Verdugo, J.M., and AlvarezBuylla, A. 2002. EGF converts transit-amplifying neurogenic precursors in the adult brain into multipotent stem cells. Neuron. 36:1021-1034

20. Nakatomi, H., et al. 2002. Regeneration of hippocampal pyramidal neurons after ischemic brain injury by recruitment of endogenous neural progenitors. Cell. 110:429-441.

21. Gundersen, H.J.G., et al. 1988. The new stereological tools: disector, fractionator, nucleator, and point sampled intercepts and their use in pathological research and diagnosis. APMIS. 96:857-881.

22. Jin, K., Mao, X.O., Sun, Y., Xie, L., and Greenberg, D.A. 2002. Stem cell factor stimulates neurogenesis in vitro and in vivo. J. Clin. Invest. 110:311-319. doi:10.1172/JCI200215251.

23. Kaser, M.R., Lakshmanan, J., and Fisher, D.A. 1992. Comparison between epidermal growth factor, transforming growth factor-alpha and EGF receptor levels in regions of adult rat brain. Brain Res. Mol. Brain Res. 16:316-322.

24. Lazar, L.M., and Blum, M. 1992. Regional distribution and developmental expression of epidermal growth factor and transforming growth factor-alpha mRNA in mouse brain by a quantitative nuclease protection assay. J. Neurosci. 12:1688-1697.

25. Nacher, J., Crespo, C., and McEwen, B.S. 2001. Doublecortin expression in the adult rat telencephalon. Eur. J. Neurosci. 14:629-644.
26. Lim, D.A., and Alvarez-Buylla, A. 1999. Interaction between astrocytes and adult subventricular zone precursors stimulates neurogenesis. Proc. Natl. Acad. Sci. U. S. A. 96:7526-7531.

27. Junier, M.P. 2000. What role(s) for TGFalpha in the central nervous system? Prog. Neurobiol. 62:443-473.

28. Song, H., Stevens, C.F., and Gage, F.H. 2002. Astroglia induce neurogenesis from adult neural stem cells. Nature. 417:39-44.

29. Steindler, D.A., and Pincus, D.W. 2002. Stem cells and neuropoiesis in the adult human brain. Lancet. 359:1047-1054

30. Cooper-Kuhn, C.M., and Kuhn, H.G. 2002. Is it all DNA repair? Methodological considerations for detecting neurogenesis in the adult brain. Brain Res. Dev. Brain Res. 134:13-21.

31. Jin, K., et al. 2001. Neurogenesis in dentate subgranular zone and rostral subventricular zone after focal cerebral ischemia in the rat. Proc. Natl. Acad. Sci. U. S. A. 98:4710-4715.

32. Koos, T., and Tepper, J.M. 1999. Inhibitory control of neostriatal projection neurons by GABAergic interneurons. Nat. Neurosci. 2:467-472.

33. Planas, A.M., Justicia, C., Soriano, M.A., and Ferrer, I. 1998. Epidermal growth factor receptor in proliferating reactive glia following transient focal ischemia in the rat brain. Glia. 23:120-129.

34. Carles, J., and Planas, A.M. 1999. Transforming growth factor-[alpha] acting at the epidermal growth factor receptor reduces infarct volume after permanent middle cerebral artery occlusion in rats. J. Cereb. Blood Flow Metab. 19:128-132.

35. Burrows, R.C., Wancio, D., Levitt, P., and Lillien, L. 1997. Response diversity and the timing of progenitor cell maturation are regulated by developmental changes in EGFR expression in the cortex. Neuron. 19:251-267.

36. Lillien, L., and Raphael, H. 2000. BMP and FGF regulate the development of EGF-responsive neural progenitor cells. Development. 127:4993-5005.

37. Lillien, L., and Wancio, D. 1998. Changes in epidermal growth factor receptor expression and competence to generate glia regulate timing and choice of differentiation in the retina. Mol. Cell Neurosci. 10:296-308.

38. Liu, Y., et al. 2001. Neuroprotective effect of treatment with human albumin in permanent focal cerebral ischemia: histopathology and cortical perfusion studies. Eur. J. Pharmacol. 428:193-201.

39. Belayev, L., Liu, Y., Zhao, W., Busto, R., and Ginsberg, M.D. 2001. Human albumin therapy of acute ischemic stroke: marked neuroprotective efficacy at moderate doses and with a broad therapeutic window. Stroke. 32:553-560.

40. Belayev, L., et al. 1999. Neuroprotective effect of high-dose albumin therapy against global ischemic brain injury in rats. Brain Res. 845:107-111.

41. Jin, K., et al. 2002. Heparin-binding epidermal growth factor-like growth factor: hypoxia-inducible expression in vitro and stimulation of neurogenesis in vitro and in vivo. J. Neurosci. 22:5365-5373.

42. Fallon, J., et al. 2000. In vivo induction of massive proliferation, directed migration, and differentiation of neural cells in the adult mammalian brain. Proc. Natl. Acad. Sci. U. S. A. 97:14686-14691.

43. Fiore, M., Triaca, V., Amendola, T., Tirassa, P., and Aloe, L. 2002. Brain NGF and EGF administration improves passive avoidance response and stimulates brain precursor cells in aged male mice. Physiol. Behav. 77:437-443.

44. Kawaguchi, Y., Wilson, C.J., Augood, S.J., and Emson, P.C. 1995. Striatal interneurones: chemical, physiological and morphological characterization. Trends Neurosci. 18:527-535.

45. Eyles, D.W., McGrath, J.J., and Reynolds, G.P. 2002. Neuronal calciumbinding proteins and schizophrenia. Schizophr. Res. 57:27-34.

46. Gernert, M., Hamann, M., Bennay, M., Loscher, W., and Richter, A. 2000 Deficit of striatal parvalbumin-reactive GABAergic interneurons and decreased basal ganglia output in a genetic rodent model of idiopathic paroxysmal dystonia. J. Neurosci. 20:7052-7058.

47. Kosinski, C.M., et al. 1999. Intranuclear inclusions in subtypes of striatal neurons in Huntington's disease transgenic mice. Neuroreport. 10:3891-3896.

48. Meade, C.A., et al. 2000. Transient global ischemia in rats yields striatal projection neuron and interneuron loss resembling that in Huntington's disease. Exp. Neurol. 166:307-323.

49. Kojima, A., and Tator, C.H. 2002. Intrathecal administration of epidermal growth factor and fibroblast growth factor 2 promotes ependymal proliferation and functional recovery after spinal cord injury in adult rats. J. Neurotrauma. 19:223-238.

50. Fricker-Gates, R.A., et al. 2000. EGF infusion stimulates the proliferation and migration of embryonic progenitor cells transplanted in the adult rat striatum. Exp. Neurol. 165:237-247.

51. Mason, H.A., Ito, S., and Corfas, G. 2001. Extracellular signals that regulate the tangential migration of olfactory bulb neuronal precursors: inducers, inhibitors, and repellents. J. Neurosci. 21:7654-7663.

52. Hillered, L., Hallstrom, A., Segersvard, S., Persson, L., and Ungerstedt, U. 1989. Dynamics of extracellular metabolites in the striatum after middle cerebral artery occlusion in the rat monitored by intracerebral microdialysis. J. Cereb. Blood Flow Metab. 9:607-616. 